\title{
Design of Accounting Information System of Modern Enterprises under Informatization Environment
}

\author{
Meng Fu \\ Zhejiang College, Shanghai University of Finance and Economics, Jinhua, 321013, China
}

Keywords: modern enterprises; accounting information system; design

\begin{abstract}
The great development of modern information technology brings new challenges to enterprises and makes the design of accounting information system of enterprises a focus issue in the field of enterprise informatization. This paper describes main contents of accounting information system of modern enterprises and discusses its design methods from two aspects hardware and software.
\end{abstract}

\section{Main contents of accounting information system of modern enterprises}

First, descriptions of accounting information resources. Information resources have gradually become a most important strategic resource of enterprises. The operation of enterprise accounting information system has significant influence on enterprise performance. Descriptions of accounting information system are mainly realized through the establishment of corresponding metadata. Modern enterprise accounting information has a wide range of users. Only workers majoring in audit and accounting can understand the meaning of accounting information published by enterprises comprehensively. Non-specialized persons only want to understand the information they consider valuable and such information must be described as the form they can understand. Therefore, data description is very important for information users. On that account, it is required to integrate high-level functions such as searching requirements of accounting information users, characteristics of accounting information resources and excavation and evaluation of system information so as to establish a metadata set that can assist enterprises in identification, description, organization and management of accounting information.

Second, collection and organization tool of accounting information. This tool platform mainly includes user registration, management and maintenance, administrator station management and recommended resources of ordinary users. Accounting information system platform mainly has two functions: first, it involves manpower, mainly including functions of different participants such as system administrator, content administrator and ordinary users. System administrator has the highest authority, mainly including management over content administrator and user information and classification of accounting information. Content administrator is mainly responsible for distributed collection, indexing, organization and release of various network information resources and examination of various resources recommended by users. Ordinary users can read existing resources in the system, recommend new resources and submit them to content administrator in the form of accounting data analysis. Meanwhile, they can implement information feedback and interaction and obtain individualized information. The other system function is automatically executed by the system, mainly including automatic monitoring on network information source and implementation of quality assessment.

Third, automatic monitoring on accounting information source and comprehensive data analysis. Data in accounting information resource library are analyzed mainly through the combination of manual review and automatic monitoring. Enterprise accounting information system can provide data obtained by automatic monitoring and analysis on automatically obtained basic index data. As accounting resources have great variability, accounting information resources collected are considerable. To ensure that various resources in accounting information system have availability and timeliness and the corresponding relationship among various accounting data has accuracy, it is required to use real-time testing of computer software for automatic analysis and meanwhile record the monitoring time and period of each object under monitoring and use the system to implement 
monitoring procedure periodically so as to obtain the monitoring result. The monitoring result should be reported to administrator in time so as to realize the function of important supervision of accounting information system.

Forth, excavation and provision of enterprise accounting information. Administrators can collect and analyze various accounting information, thus improving the accuracy and depth of accounting resources. However, types and categories of requirements of modern enterprises for accounting information are changing continuously and the model tool used to analyze accounting information is also updated. Therefore, it is required to implement data excavation for enterprise accounting information, mainly including the following steps: first, understand the problem area; second, prepare relevant data subset; then, implement data excavation; finally, apply achievements of data excavation. Data excavation can give fully play to specialties of human and computer, thus reducing the workload of administrators and improving the depth and breadth of accounting information in the system.

Fifth, information feedback and interaction of enterprise accounting information users. The ultimate goal of enterprise accounting information system is to help users use various accounting resources more fully and effectively, thus serving different information requirements of users. Information interaction between users and the system and among users can play a vital role. The system can mainly provide online user survey, message, bulletin board system and online discussion etc. Except the way that users provide information actively and positively, the system also implements automatic collection, organization and analysis of effective information provided by users when customers use various functions and resources of the system so as to understand users' requirements for relevant accounting information and provide individualized services for collecting corresponding information.

\section{Hardware design methods of accounting information system of modern enterprises}

\section{(I) Stand-alone structure design method}

The main feature of this design method is that each computer should be supplied with a piece of corresponding external equipment in this structure so as to handle various different accounting information businesses respectively and they are not connected directly and maintain their own independence. In enterprise accounting information system, once any accounting information needs to be delivered, it is required to use the form of copy on USB flash disk. Its advantages are that multiple concurrent computers have strong independence, good privacy of accounting data, high stability, simple configuration and management and low cost. Its disadvantage is that each computer can only be used by one user, which causes low overall efficiency of this system, as computers do not directly produce data exchange relation and hardly have data sharing performance. This design method applies to units without local area network. Currently, it is a design structure used more frequently in accounting information system of small- and medium-sized enterprises.

\section{(II) Multi-user structure design method}

The main feature of this design method is that the same host computer is connected to multiple different terminals in this structure. As a computer with the best performance in the system, host computer can store all applications and databases in this system so as to handle and store data intensively. The terminal of this equipment does not have CPU or external memory. It is only supplied with input and output equipment. In terms of implementation, this system is a diskless workstation. They consider host computer as a big disk for sharing. Only by operating corresponding procedures stored in host computer can the terminal access corresponding data stored in host computer well. Its advantage is that it can solve bottlenecks of data input and output and realize the sharing of various databases. Its disadvantage is that, under the condition of large size of application software and high data volume, it is required to not only transfer corresponding procedures, but also deliver relevant documents, thus causing high load of this system and system obstruction and application collision and influencing the operating efficiency of accounting information system. If host computer has any fault, all terminals cannot continue to work, thus causing breakdown of the whole system. Meanwhile, this system has a poor expansion performance, 
low number of application software and high maintenance cost. This system mainly applies to enterprises with large amount of accounting business, centralized data processing and great ability of system maintenance. Currently, it is used more in financial industry and post and telecommunication industry.

\section{(III) Network structure design method}

The main feature of this design method is that multiple computers scattered in different geographic locations are connected via network equipment so that computers can realize direct data exchange and resource sharing. It can be divided into two categories - client/server and browser/server according to the way of operation and data transmission. First, client/server structure design method. Its main feature is that $\mathrm{C} / \mathrm{S}$ structure is composed of multiple clients and one or more servers and relevant network communication equipment. This structure is developing towards multilevel structure and is an ideal choice for the design of accounting information system of large and medium enterprises. Its working principle is that effective distribution of applications and tasks is implemented between server and client and system application is divided into two constituent parts - front client and background server. The former is mainly used for sharing resource management application, which can implement respective application processing and share various abundant resources provided by server. The latter is mainly oriented at users and pays attention to data access security, backup and recovery and implementation of retrieval and indexing. Its advantage is that this structure no longer needs to transmit all, thus reducing the load of transmission, and that server can share various resources such as software and data intensively for the convenience of management and monitoring. Its disadvantage is that the territorial scope of sharing is very limited. Second, browser/server design method. Its main feature is that it is established based on international internet or enterprise intranet and it is an applied mode and the most advanced network structure with the greatest development potential. It applies to large transnational and trans-regional group enterprises. Its working principle is that $\mathrm{B} / \mathrm{S}$ structure integrates network functions and is supplied with electric energy of Web browser so as to query relevant information in Web browser. Server can continue to be broken into application server and various databases, documents and host server.

\section{(IV) Cloud computing structure design method}

Cloud computing is the development of distributed processing, parallel processing and grid computing under modern informatization conditions and an emerging commercial supercomputing mode under network environment. In hardware design, infrastructure as a service (IAAS) is advocated under cloud computing environment. Infrastructure mainly includes server, network resources and data pool. Facilities above are provided for customers for use as needed through service. Service supplier completely has the ownership of facilities involved in accounting information system and is responsible for daily operation of machine room. It is thus clear that it is unnecessary to buy expensive facilities in the design of enterprise accounting information system under this mode and only required computing power and storage capacity need to be rented.

\section{Software design methods of accounting information system of modern enterprises}

\section{(I) Industrial application structure design method}

The main business of industrial enterprises is production and management. They belong to social economic organizations and their main feature is that production and management are composed of three different links which are connected organically - supply, production and sales. Their distinct characteristic is that processing and production links exist and enterprises use reproduction to realize profits. In the process of implementing reproduction, enterprises should start from monetary capital form and use three different stages - purchasing, production and sales successively to realize appreciation of monetary capital. Therefore, industrial enterprise accounting not only contains financial accounting, but also includes cost control management during production and sales management in the last stage. To meet requirements of industrial enterprises in accounting treatment, 


\section{(II) Business application structure design method}

Commercial enterprises are economic organizations in the category of commodity circulation and operation aiming at profiting. Their main feature is the combination of wholesale and retail. Commodities are often subject to batch management. Consignment business exists and different departments check various expenditures independently. Generally, it is necessary to use commodity varieties for statistics of relevant purchasing, sales and storage data. Suppliers need to count the quantity of stock replenished and payables. The sales volume and receivables should be counted according to trade debtors. It is required to prepare accounting statement at the end of the term and submit it to the competent department in time. Considering the rapid promotion and application of modern network technology in business field, business enterprises are faced with new challenges such as restructuring and reconstruction. This causes the emergence of many large trans-regional business enterprises. More demands and higher requirements are put forward for the design of accounting information system of business enterprises. Currently, business enterprises have two design methods available: first, basic application structure, which pays attention to business accounting and management of capital flow of business enterprises; second, extended application structure, which pays attention to integrated management of purchasing, sales and storage of business enterprises.

\section{(III) Cloud computing structure design method}

Software design under cloud computing environment advocates software as service (SAAS). SAAS tends to be mature with the development of network technology and is a new software design method emerging at the start of this century. Software service provider installs application software in infrastructure and is responsible for all preliminary operation and later management and maintenance. Under this mode, accounting management system, checking system, decision-making system and other application modules closely related to accounting information system are installed in cloud. Customers can manage accounting activities of enterprises at any time as long as they order the corresponding business module. They don't need to purchase the usage license of financial software and maintain it. The relationship between software supplier and customers is changed into the relationship of on-demand service from one-off deal relation.

\section{Conclusion}

In conclusion, modern enterprises should select different hardware and software design methods according to their own scale, industrial characteristics, demand and strategic development plan in the process of designing accounting information system. Only in this way can the design of accounting information system of modern enterprises shoot the arrow at the target and the effectiveness of this accounting information system be maximized.

\section{References:}

[1] Huang Cuiling. Research on Design of Enterprise Accounting Information Management System - Based on Perspective of Corporate Governance [J]. Chinese Collective Economy, 2012(22).

[2] Li Jingsi. Research on Design of Internal Control of Enterprise Accounting Information System under ERP Environment [J]. Business Accounting, 2012(24).

[3] Zhang Yinping. Analysis on Reasons Influencing Internal Control of Enterprise Accounting Information System [J]. Modern Business, 2013(29).

[4] Li Shanying. Brief Analysis on Enterprise Accounting Information System [J]. Economic Research Guide, 2013(35).

[5] Pan Xiamin. Research on Influencing Factors of Internal Control of Enterprise Accounting Information System [J]. Management Administrator, 2014(5). 